\title{
ENTROPY AND THE UNIFORM MEAN ERGODIC THEOREM FOR A FAMILY OF SETS
}

\author{
TERRENCE M. ADAMS AND ANDREW B. NOBEL
}

\begin{abstract}
We define a notion of entropy for an infinite family $\mathcal{C}$ of measurable sets in a probability space. We show that the mean ergodic theorem holds uniformly for $\mathcal{C}$ under every ergodic transformation if and only if $\mathcal{C}$ has zero entropy. When the entropy of $\mathcal{C}$ is positive, we establish a strong converse showing that the uniform mean ergodic theorem fails generically in every isomorphism class, including the isomorphism classes of Bernoulli transformations. As a corollary of these results, we establish that every strong mixing transformation is uniformly strong mixing on $\mathcal{C}$ if and only if the entropy of $\mathcal{C}$ is zero, and obtain a corresponding result for weak mixing transformations.
\end{abstract}

\section{INTRODUCTION}

Let $T$ be an ergodic measure preserving transformation of a Lebesgue probability space $(\mathcal{X}, \mathcal{S}, \mu)$. For any fixed measurable set $C$, the ergodic theorem guarantees the pointwise and mean convergence of the relative frequency $n^{-1} \sum_{i=0}^{n-1} I_{C}\left(T^{i} x\right)$ to $\mu(C)$. In many cases of theoretical and practical interest, it is useful (or sometimes essential) to know that the ergodic theorem holds uniformly over an infinite family $\mathcal{C}$ of measurable sets. A uniform pointwise ergodic theorem holds for a family $\mathcal{C}$ if

$$
\sup _{C \in \mathcal{C}}\left|\frac{1}{n} \sum_{i=0}^{n-1} I_{C}\left(T^{i} x\right)-\mu(C)\right| \rightarrow 0 \text { with probability one as } n \text { tends to infinity. }
$$

In probability and statistics, the trajectory $\left\{T^{i} x: i \geq 0\right\}$ in 11 is replaced by a stationary sequence $\left\{X_{i}: i \geq 0\right\}$ of random variables; trajectories constitute the special case in which $X_{i}=T^{i} X_{0}$ where $X_{0}$ has distribution $\mu$. Uniform pointwise ergodic theorems, also known as Glivenko-Cantelli theorems, have been widely studied in the machine learning and empirical process literature, most often in the case of independent identically distributed (i.i.d.) sequences of random variables; results and further references can be found in [12, 6, 14]. There is also a companion literature concerning uniform pointwise ergodic theorems for dependent sequences of random variables, including the case of trajectories. Adams and Nobel [2] established that (1) holds for every ergodic measure preserving transformation $T$ of a probability space $(\mathcal{X}, \mathcal{S}, \mu)$ provided that the family $\mathcal{C}$ has finite Vapnik-Chervonenkis (VC) dimension. (The definition of VC dimension is given in Section 1.1 below.) Extensions

Date: March 2014.

2010 Mathematics Subject Classification. Primary 37A25; Secondary 60F05,37A35,37A50.

The work of Andrew Nobel was supported by NSF Grants DMS-0907177 and DMS-1310002. 
and related results can be found in von Handel [13]; see [2, 1, 13] and the references therein for more details.

The focus of this paper is uniform mean ergodic theorems. A uniform mean ergodic theorem holds for a family of measurable sets $\mathcal{C}$ if

$$
\sup _{C \in \mathcal{C}} \int\left|\frac{1}{n} \sum_{i=0}^{n-1} I_{C}\left(T^{i} x\right)-\mu(C)\right| d \mu \rightarrow 0 \text { as } n \text { tends to infinity. }
$$

Note that (1) clearly implies (2) but not vice versa. For any i.i.d. sequence of random variables, elementary arguments show that the uniform mean ergodic theorem holds for any family $\mathcal{C}$ of measurable sets, and the same holds true under the type mixing conditions typically imposed in statistical problems. Thus uniform mean ergodic theorems are primarily of interest for trajectories and related strongly dependent processes.

We establish an equivalence between uniform mean ergodic theorems for measure preserving transformations of $(\mathcal{X}, \mathcal{S}, \mu)$ and the complexity of the family $\mathcal{C}$. The complexity of $\mathcal{C}$ is quantified in terms of an entropy measure that we now define. Given measurable sets $C_{1}, \ldots, C_{n} \in \mathcal{S}$, let $\vee_{i=1}^{n} C_{i}$ denote their join, or equivalently, the partition $\Pi$ of $\mathcal{X}$ having cells of the form $\tilde{C}_{1} \cap \cdots \cap \tilde{C}_{n}$ where each $\tilde{C}_{i} \in\left\{C_{i}, C_{i}^{c}\right\}$. The join $\Pi_{0} \vee \Pi_{1}$ of two partitions is defined to be the join of their constituent cells; thus $\Pi_{0} \vee \Pi_{1}$ refines $\Pi_{0}$ and $\Pi_{1}$. Recall that the entropy of finite measurable partition $\Pi$ of $\mathcal{X}$ is defined by

$$
H_{\mu}(\Pi)=-\sum_{A \in \Pi} \mu(A) \log \mu(A) .
$$

Here and in what follows we assume, without loss of generality, that all logarithms are base 2 , and that each cell of a partition has positive measure. Let $\mathcal{C} \subseteq \mathcal{S}$ be a family of measurable subsets of $\mathcal{X}$. For every ordered sequence $\mathbb{S}=\left(C_{1}, C_{2}, \ldots\right)$ with $C_{i} \in \mathcal{C}$ let

$$
H_{\mu}(\mathbb{S})=\liminf _{n \rightarrow \infty} \frac{1}{n} H_{\mu}\left(\vee_{i=1}^{n} C_{i}\right) .
$$

The entropy of the family $\mathcal{C}$ is defined by

$$
H_{\mu}(\mathcal{C})=\sup H_{\mu}(\mathbb{S})
$$

where the supremum is over all ordered sequences $\mathbb{S}$ of sets in $\mathcal{C}$. Our principal result is the following theorem.

Theorem 1. Let $(\mathcal{X}, \mathcal{S}, \mu)$ be a Lebesgue probability space, and let $\mathcal{C} \subseteq \mathcal{S}$ be a family of measurable subsets of $\mathcal{X}$. Then $H_{\mu}(\mathcal{C})=0$ if and only if (2) holds for every ergodic measure preserving transformation $T$ of $(\mathcal{X}, \mathcal{S}, \mu)$.

The only-if part of Theorem 1 follows from elementary approximation properties of zero entropy families, and is valid for any probability space. The converse part of the theorem requires us to exhibit a $\mu$-preserving ergodic tranformation $T$ for which $(2)$ fails when $H_{\mu}(\mathcal{C})$ is positive. A stronger, generic, version of the converse is described below. 
Remark: Theorem 1 may also be expressed in the terminology of stochastic processes. Let $(\mathcal{X}, \mathcal{S}, \mu)$ be a Lebesgue probability space and let $\mathcal{C} \subseteq \mathcal{S}$ be a family of measurable subsets of $\mathcal{X}$. It follows from Theorem 1 that $H_{\mu}(\mathcal{C})=0$ if and only if

$$
\sup _{C \in \mathcal{C}}\left|\frac{1}{n} \sum_{i=0}^{n-1} I_{C}\left(U_{i}\right)-\mu(C)\right| \rightarrow 0 \text { with probability one as } n \rightarrow \infty
$$

for every stationary ergodic process $U_{0}, U_{1}, \ldots$ taking values in $(\mathcal{X}, \mathcal{S})$ and such that $U_{i}$ has distribution $\mu$.

\subsection{Examples of Zero Entropy Families.}

Finite dimensional families. The (dual) Vapnik-Chervonenkis (VC) dimension of a family $\mathcal{C}$ is the largest $k \geq 1$ for which there exists sets $C_{1}, \ldots, C_{k} \in \mathcal{C}$ whose join $\vee_{i=1}^{k} C_{i}$ has cardinality $2^{k}$, the largest possible value for $k$ sets. If $\mathcal{C}$ contains arbitrary large finite collections with maximal joins, then its dimension is infinite. It is known (cf. [12]) that if $\mathcal{C}$ has finite VC-dimension then for $C_{1}, \ldots, C_{n} \in \mathcal{C}$ the cardinality of $\vee_{i=1}^{n} C_{i}$ is bounded by a polynomial in $n$ that depends only on the dimension. It then follows from the elementary bound $H_{\mu}(\Pi) \leq \log |\Pi|$ that any family $\mathcal{C}$ with finite VC-dimension has entropy zero for all measures $\mu$. Although it is considerably more indirect, one may reach the same conclusion by combining Theorem 1 with the principal result of [2]. For general $\mathcal{X}$, the family of sets $\{x: g(x) \geq 0\}$ where $g$ ranges over a finite dimensional vector space of real valued functions on $\mathcal{X}$ has finite $\mathrm{VC}$ dimension. For $\mathcal{X}=\mathbb{R}^{d}$, families with finite $\mathrm{VC}$ dimension include convex polytopes with at most $k$ faces and the family of open balls (with arbitrary center and radius). See [12] and [6] for more details. Note that zero entropy families may have infinite dimension: it is easy to construct $\mathcal{C}=\left\{C_{1}, C_{2}, \ldots\right\}$ with infinite VC dimension such that $\mu\left(C_{i}\right) \rightarrow 0$.

Bracketing families. A family $\mathcal{C}$ is said to be bracketing with respect to a measure $\mu$ if for every $\delta>0$ there exists a finite family $\mathcal{D}$ such that for each $C \in \mathcal{C}$ there exist sets $U, V \in \mathcal{D}$ such that $U \subseteq C \subseteq V$ and $\mu(V \backslash U)<\delta$. If $\mathcal{C}$ is bracketing with respect to $\mu$ one may readily show using the characterization of zero-entropy families in Lemma 3 below that $H_{\mu}(\mathcal{C})=0$. Alternatively, if $\mathcal{C}$ is bracketing with respect to $\mu$ then it is easy to show that the uniform pointwise ergodic theorem (1) holds for every $\mu$-preserving transformation of $(\mathcal{X}, \mathcal{S})$ and therefore $H_{\mu}(\mathcal{C})=0$ by Theorem 1 . If $\mathcal{X}=\mathbb{R}^{d}$ and $\mu$ is absolutely continuous with respect to Lebesgue measure then the family of convex subsets of $\mathcal{X}$ is bracketing with respect to $\mu$.

Clustered families. It is easy to see that a family $\mathcal{C}$ has entropy $H_{\mu}(\mathcal{C})=0$ if there is a fixed measurable set $C_{0}$ such that for each $\delta>0$ at most finitely many sets in $\mathcal{C}$ satisfy $\mu\left(C \triangle C_{0}\right)>\delta$. 
Stability Properties. Zero entropy families are closed under natural set theoretic operations. A finite union of zero entropy families has zero entropy. If families $\mathcal{C}_{1}$ and $\mathcal{C}_{2}$ have zero entropy, then so too do the families of complements $\left\{C^{c}: C \in \mathcal{C}_{1}\right\}$, unions $\left\{C_{1} \cup C_{2}: C_{1} \in \mathcal{C}_{1}, C_{2} \in \mathcal{C}_{2}\right\}$, and intersections $\left\{C_{1} \cap C_{2}: C_{1} \in \mathcal{C}_{1}, C_{2} \in \mathcal{C}_{2}\right\}$.

Remark: Recall that the entropy of a fixed measure preserving transformation $T$ is defined by

$$
H_{\mu}(T):=\sup _{\Pi} \lim _{n \rightarrow \infty} n^{-1} H\left(\vee_{i=0}^{n-1} T^{-i} \Pi\right),
$$

where the supremum is taken over all finite measurable partitions $\Pi$ of $\mathcal{X}$. We note that the definitions of $H_{\mu}(\mathcal{C})$ and $H_{\mu}(T)$ do not coincide: in (3) we scale by the number of sets, equivalently two-way partitions, that give rise to the join, while in (4) one scales according to the number of iterates of $T^{-1}$, without regard to the cardinality of the partition $\pi$.

1.2. Strong Converse and Corollaries. Let $\Phi$ be the space of invertible measure preserving transformations of $(\mathcal{X}, \mathcal{S}, \mu)$ endowed with the weak topology, namely $\phi_{r} \rightarrow \phi$ if, for all measurable sets $A \in \mathcal{S}$,

$$
\lim _{r \rightarrow \infty} \mu\left(\phi_{r} A \triangle \phi A\right)=0
$$

Each $\phi \in \Phi$ represents an equivalence class $[\phi]=\{\psi \in \Phi: \mu(\phi=\psi)=1\}$ of transformations; for convenience of notation we identify $[\phi]$ with $\phi$. Let $\phi \psi=\phi \circ \psi$ denote the composition of transformations $\phi, \psi \in \Phi$. For a measure preserving transformation $T$ and $\phi \in \Phi$, let $T_{\phi}$ denote the conjugate transformation $\phi^{-1} \circ T \circ \phi$.

Theorem 2. Let $T$ be an invertible ergodic measure preserving transformation of a Lebesgue probability space $(\mathcal{X}, \mathcal{S}, \mu)$. If $\mathcal{C}$ is a class of measurable sets in $\mathcal{X}$ such that $H(\mathcal{C})>0$, then there exists a dense $G_{\delta}$ set $G \subset \Phi$ such that for each $\phi \in G$,

$$
\limsup _{n \rightarrow \infty} \sup _{C \in \mathcal{C}} \int\left|\frac{1}{n} \sum_{i=0}^{n-1} I_{C}\left(T_{\phi}^{i} x\right)-\mu(C)\right| d \mu>0 .
$$

Recall that a measure preserving transformation $T$ of $(\mathcal{X}, \mathcal{S}, \mu)$ is strong mixing (and therefore ergodic) if for all measurable sets $A, B \in \mathcal{S}$,

$$
\lim _{n \rightarrow \infty}\left|\mu\left(A \cap T^{-n} B\right)-\mu(A) \mu(B)\right|=0 .
$$

As a corollary of Theorems 1 and 2 we obtain an entropy based characterization of uniform strong mixing. An analogous result for weak mixing transformations is given in Corollary 2 of Section 4.1 .

Corollary 1. Let $T$ be a strong mixing transformation of $(\mathcal{X}, \mathcal{S}, \mu)$ and let $\mathcal{C} \subseteq \mathcal{S}$. 
(i) If $H(\mathcal{C})=0$ then $T$ is uniform strong mixing on $\mathcal{C}$, in the sense that

$$
\lim _{n \rightarrow \infty} \sup _{A, B \in \mathcal{C}}\left|\mu\left(A \cap T^{-n} B\right)-\mu(A) \mu(B)\right|=0 .
$$

(ii) Suppose that $T$ is invertible. If $H(\mathcal{C})>0$, then there exists a dense $G_{\delta}$ set $G \subset \Phi$ such that for each $\phi \in G$,

$$
\limsup _{n \rightarrow \infty} \sup _{C \in \mathcal{C}}\left|\mu\left(C \cap T_{\phi}^{-n} C\right)-\mu(C)^{2}\right|>0 \text {. }
$$

Note that if $T \in \Phi$ is strong mixing then $T_{\phi}$ is strong mixing as well. As a counterpoint to part (ii) of Corollary 1, we show that for positive entropy families uniform mixing need not fail for every strong mixing transformation. Suppose $S$ and $T$ are invertible commuting transformations on $(\mathcal{X}, \mathcal{S}, \mu)$ that form a strong mixing $\mathbb{Z}^{2}$-action in the sense that

$$
\mu\left(T^{n} S^{m} A \cap B\right) \rightarrow \mu(A) \mu(B)
$$

for all $A, B \in \mathcal{S}$ as $|m|+|n| \rightarrow \infty$. (See [4, 7, 10] for explicit constructions of strong mixing $\mathbb{Z}^{2}$-actions.) Let $C \in \mathcal{S}$ be such that $\mu(C)=\frac{1}{2}$. Define $C_{i}=S^{i} C$ and let $\mathcal{C}=\left\{C_{i}: i \in \mathbb{N}\right\}$. Since $S$ is strong mixing, $H(\mathcal{C})=\log 2>0$. On the other hand,

$$
\mu\left(T^{n} C_{i} \cap C_{j}\right)=\mu\left(T^{n} S^{i} C \cap S^{j} C\right)=\mu\left(T^{n} S^{i-j} C \cap C\right) \rightarrow \mu(C)^{2}
$$

as $|n|+|i-j| \rightarrow \infty$. Thus

$$
\lim _{n \rightarrow \infty} \sup _{i, j}\left|\mu\left(T^{n} C_{i} \cap C_{j}\right)-\mu\left(C_{i}\right) \mu\left(C_{j}\right)\right|=0
$$

so that $T$ is uniformly mixing over the collection $\mathcal{C}$.

1.3. Overview of the Paper. The next section is devoted to several elementary approximation properties of entropy. Section 3 contains the proofs of Theorems 1 and 2 . The proof of Corollary 1 and the statement of an analogous result for weak mixing are given in Section 4 .

\section{Basic Properties of Entropy}

Let $(\mathcal{X}, \mathcal{S}, \mu)$ be a probability space. (The Lebesgue assumption is not necessary for the results of this section.) If $\Pi_{0}, \Pi_{1}$ are finite measurable partitions of $\mathcal{X}$, the conditional entropy of $\Pi_{1}$ given $\Pi_{0}$ is defined by

$$
H_{\mu}\left(\Pi_{1} \mid \Pi_{0}\right)=-\sum_{A \in \Pi_{0}} \mu(A) \sum_{B \in \Pi_{1}} \frac{\mu(B \cap A)}{\mu(A)} \log \frac{\mu(B \cap A)}{\mu(A)} .
$$

For $C \in \mathcal{S}$ define $H_{\mu}(C \mid \Pi)=H_{\mu}\left(\left\{C, C^{c}\right\} \mid \Pi\right)$. If $\mathcal{D}$ is a collection of measurable sets, define $\cup \mathcal{D}=\cup_{A \in \mathcal{D}} A$. In the remainder of this section the measure $\mu$ is fixed: in order to reduce notation we will drop the subscript from the entropy. 
The importance of entropy in our analysis derives from its connection with the approximation of sets by finite partitions. We present two elementary approximation results, and an alternative characterization of zero entropy families, whose proofs are provided for completeness.

Lemma 1. For every $\epsilon>0$, there exists $\delta>0$ such that for every finite measurable partition $\Pi$ and every measurable set $C$ the relation $H(C \mid \Pi)>\epsilon$ implies

$$
\mu(\cup\{A \in \Pi: \delta \mu(A) \leq \mu(A \cap C) \leq(1-\delta) \mu(A)\})>\delta .
$$

Proof: Fix $\epsilon>0$ and let $0<\delta<\epsilon / 2$ be such that $-x \log x<\epsilon / 4$ when $0<x<\delta$ or $1-\delta<x<1$. Let $\Pi$ be a finite measurable partition and let $C$ be a measurable set such that $H(C \mid \Pi)>\epsilon$. Define the collections

$$
\Pi_{1}=\{A \in \Pi: \mu(A \cap C)<\delta \mu(A)\} \text { and } \Pi_{2}=\{A \in \Pi: \mu(A \cap C)>(1-\delta) \mu(A)\} .
$$

Let $\Pi_{3}=\Pi \backslash\left(\Pi_{1} \cup \Pi_{2}\right)$. The definition of $\delta$ and the fact that $H\left(\left\{B, B^{c}\right\}\right) \leq 1$ for any measure $\mu$ together imply that

$$
\begin{aligned}
H(C \mid \Pi)= & -\sum_{A \in \Pi_{3}} \mu(A)\left[\frac{\mu(A \cap C)}{\mu(A)} \log \frac{\mu(A \cap C)}{\mu(A)}+\frac{\mu\left(A \cap C^{c}\right)}{\mu(A)} \log \frac{\mu\left(A \cap C^{c}\right)}{\mu(A)}\right] \\
& -\sum_{A \in \Pi_{3}^{c}} \mu(A)\left[\frac{\mu(A \cap C)}{\mu(A)} \log \frac{\mu(A \cap C)}{\mu(A)}+\frac{\mu\left(A \cap C^{c}\right)}{\mu(A)} \log \frac{\mu\left(A \cap C^{c}\right)}{\mu(A)}\right] \\
< & \mu\left(\cup \Pi_{3}\right)+\frac{\epsilon}{2} \mu\left(\cup \Pi_{3}^{c}\right) \leq \mu\left(\cup \Pi_{3}\right)+\frac{\epsilon}{2} .
\end{aligned}
$$

As $H(C \mid \Pi)>\epsilon$, it follows that $\mu\left(\cup \Pi_{3}\right)>\epsilon / 2>\delta$.

Lemma 2. For every $\epsilon>0$, there exists $\delta>0$ such that for every finite measurable partition $\Pi$ and every measurable set $C$ satisfying $H(C \mid \Pi)<\delta$ there is a subcollection $\Pi_{0} \subset \Pi$ depending on $C$ such that $\mu\left(\left(\cup \Pi_{0}\right) \triangle C\right)<\epsilon$.

Proof: Fix $\epsilon>0$ and let $\alpha=-(\epsilon / 4) \log (\epsilon / 4)-(1-\epsilon / 4) \log (1-\epsilon / 4)$. Choose $0<\delta<\epsilon \alpha / 4$, and let $\Pi$ and $C$ be such that $H(C \mid \Pi)<\delta$. Define the collections

$$
\Pi_{0}=\{A \in \Pi: \mu(A \cap C)>(1-\epsilon) \mu(A) / 4\} \text { and } \Pi_{1}=\{A \in \Pi: \mu(A \cap C)<\epsilon \mu(A) / 4\} .
$$

Let $\Pi_{2}=\Pi \backslash\left(\Pi_{0} \cup \Pi_{1}\right)$ and define $B_{i}=\cup \Pi_{i}$. Note that

$$
H(C \mid \Pi) \geq \sum_{A \in \Pi_{2}} \mu(A) \alpha=\alpha \mu\left(B_{2}\right),
$$

and therefore $\mu\left(B_{2}\right)<\delta / \alpha<\epsilon / 4$. The definition of $\Pi_{1}$ ensures that $\mu\left(C \cap B_{1}\right)<\epsilon / 4$, so that

$$
\mu\left(C \backslash B_{0}\right)=\mu\left(C \cap B_{1}\right)+\mu\left(C \cap B_{2}\right)<\frac{\epsilon}{4}+\frac{\epsilon}{4}=\frac{\epsilon}{2} .
$$

Finally, the definition of $\Pi_{0}$ ensures that $\mu\left(B_{0} \backslash C\right)<\epsilon / 4$, and the result follows. 
Lemma 3. Let $\mathcal{C} \subseteq \mathcal{S}$ be a family of measurable subsets of $X$. The entropy $H(\mathcal{C})=0$ if and only if for each $\delta>0$ there exists a finite measurable partition $\Pi$ such that $H(C \mid \Pi) \leq \delta$ for all $C \in \mathcal{C}$.

Proof: The proof follows from standard facts about entropy. Suppose that the approximation condition in the statement of the lemma holds. Let $\delta>0$ and let $\mathbb{S}=\left(C_{1}, C_{2}, \ldots\right)$ be an ordered sequence of sets in $\mathcal{C}$. For each $n \geq 1$ define $\Pi_{n}=\vee_{i=1}^{n} C_{i}$. By standard results,

$$
H\left(\Pi_{n} \mid \Pi\right) \leq \sum_{i=1}^{n} H\left(C_{i} \mid \Pi\right) \quad \text { and } \quad H\left(\Pi_{n} \mid \Pi\right)=H\left(\Pi_{n} \vee \Pi\right)-H(\Pi)
$$

Let $\Pi$ be a finite partition such that $H(C \mid \Pi)<\delta$ for all $C \in \mathcal{C}$. Then

$$
\begin{aligned}
\frac{1}{n} H\left(\vee_{i=1}^{n} C_{i}\right) & =\frac{1}{n} H\left(\Pi_{n}\right) \leq \frac{1}{n} H\left(\Pi_{n} \vee \Pi\right)=\frac{1}{n}\left[H\left(\Pi_{n} \mid \Pi\right)+H(\Pi)\right] \\
& \leq \frac{1}{n} \sum_{i=1}^{n} H\left(C_{i} \mid \Pi\right)+\frac{H(\Pi)}{n} \leq \delta+\frac{H(\Pi)}{n}<2 \delta
\end{aligned}
$$

when $n$ is sufficiently large. Since $\delta$ and $\mathbb{S}$ were arbitrarily, $H(\mathcal{C})=0$.

Now suppose that the approximation condition fails to hold. Then there exists $\delta>0$ such that for any finite partition $\Pi$ there is a set $C \in \mathcal{C}$ such that $H(C \mid \Pi) \geq \delta$. Let $C_{1}$ be any set in $\mathcal{C}$. For each $i \geq 2$ choose $C_{i} \in \mathcal{C}$ such that $H\left(C_{i}, \mid \Pi_{i-1}\right) \geq \delta$, where $\Pi_{r}=\vee_{j=1}^{r} C_{j}$. By the chain rule for entropy,

$$
H\left(\Pi_{n}\right)=\sum_{i=2}^{n} H\left(C_{i} \mid \Pi_{i-1}\right)+H\left(\Pi_{1}\right) \geq(n-1) \delta .
$$

Thus $H(\mathbb{S}) \geq \delta$ where $\mathbb{S}=\left(C_{1}, C_{2}, \ldots\right)$, and it follows that $H(\mathcal{C}) \geq \delta$.

Definition: Fix $k \geq 1$ and let $\mathcal{S}^{k}$ be $k$-fold Cartesian product of the sigma field $\mathcal{S}$. A sequence of functions $f_{n}: \mathcal{S}^{k} \rightarrow \mathbb{R}, n \geq 1$, is uniformly continuous with respect to $\mu$ if for each $\epsilon>0$ there exists a $\delta>0$ such that $A_{1}, \ldots, A_{k}, B_{1}, \ldots, B_{k} \in \mathcal{S}$ and $\mu\left(A_{i} \triangle B_{i}\right)<\delta$ for $i=1,2, \ldots, k$ imply $\left|f_{n}\left(A_{1}, \ldots, A_{k}\right)-f_{n}\left(B_{1}, \ldots, B_{k}\right)\right|<\epsilon$ for all $n \geq 1$.

Proposition 1. Let the functions $f_{n}: \mathcal{S}^{k} \rightarrow \mathbb{R}, n \geq 1$, be a uniformly continuous with respect to $\mu$ and such that $\lim _{n \rightarrow \infty} f_{n}\left(A_{1}, \ldots, A_{k}\right)=0$ for all $A_{1}, \ldots, A_{k} \in \mathcal{S}$. If $\mathcal{C} \subseteq \mathcal{S}$ is a class of measurable sets such that $H_{\mu}(\mathcal{C})=0$, then

$$
\lim _{n \rightarrow \infty} \sup _{C_{1}, \ldots, C_{k} \in \mathcal{C}} f_{n}\left(C_{1}, \ldots, C_{k}\right)=0 .
$$

Proof: Suppose that $H(\mathcal{C})=0$. Fix $\epsilon>0$ and let $\delta>0$ be as in the definition of uniform continuity above. Combining Lemma 3 and Lemma 2, we see that there exists a finite measurable partition $\Pi$ such that for every $C \in \mathcal{C}$ there is an approximating set $\hat{C} \in \mathcal{S}(\Pi)$ such that $\mu(C \triangle \hat{C})<\delta$. Here $\mathcal{S}(\Pi)$ is the (finite) family of all finite unions of cells of $\Pi$. Let 
$N \geq 1$ be so large that $\left|f_{n}\left(B_{1}, \ldots, B_{k}\right)\right| \leq \epsilon$ for every $n \geq N$ and every $B_{1}, \ldots, B_{k} \in \mathcal{S}(\Pi)$. Then for each $n \geq N$, and each sequence of sets $C_{1}, C_{2}, \ldots, C_{k} \in \mathcal{C}$,

$$
\left|f_{n}\left(C_{1}, \ldots, C_{k}\right)\right| \leq\left|f_{n}\left(C_{1}, \ldots, C_{k}\right)-f_{n}\left(\hat{C}_{1}, \ldots, \hat{C}_{k}\right)\right|+\left|f_{n}\left(\hat{C}_{1}, \ldots, \hat{C}_{k}\right)\right| \leq 2 \epsilon .
$$

As $\epsilon>0$ and the sets $C_{1}, \ldots, C_{k}$ were arbitrary, the proof is complete.

\section{Proof of Theorems 1 And 2}

\subsection{Sufficiency of Zero Entropy.}

Proposition 2. Let $(\mathcal{X}, \mathcal{S}, \mu)$ be a probability space, and let $\mathcal{C} \subseteq \mathcal{S}$. If $H_{\mu}(\mathcal{C})=0$ then for every ergodic measure preserving transformation $T$ of $(X, \mathcal{S}, \mu)$,

$$
\sup _{C \in \mathcal{C}} \int\left|\frac{1}{n} \sum_{i=0}^{n-1} I_{C}\left(T^{i} x\right)-\mu(C)\right| d \mu \rightarrow 0
$$

as $n$ tends to infinity.

Proof: For each $n \geq 1$ define the set function $f_{n}: \mathcal{S} \rightarrow \mathbb{R}$ by

$$
f_{n}(A)=\int\left|\frac{1}{n} \sum_{i=0}^{n-1} I_{A}\left(T^{i} x\right)-\mu(A)\right| d \mu .
$$

The mean ergodic theorem ensures that $f_{n}(A) \rightarrow 0$ for each $A \in \mathcal{S}$. Let $A, B$ be measurable sets. By standard arguments,

$$
\begin{aligned}
f_{n}(A)-f_{n}(B) & =\int\left(\left|\frac{1}{n} \sum_{i=0}^{n-1} I_{A}\left(T^{i} x\right)-\mu(A)\right|-\left|\frac{1}{n} \sum_{i=0}^{n-1} I_{B}\left(T^{i} x\right)-\mu(B)\right|\right) d \mu \\
& \leq \int\left|\left(\frac{1}{n} \sum_{i=0}^{n-1} I_{A}\left(T^{i} x\right)-\frac{1}{n} \sum_{i=0}^{n-1} I_{B}\left(T^{i} x\right)\right)+\mu(B)-\mu(A)\right| d \mu \\
& \leq \frac{1}{n} \sum_{i=0}^{n-1} \int_{X}\left|I_{A}\left(T^{i} x\right)-I_{B}\left(T^{i} x\right)\right| d \mu+|\mu(B)-\mu(A)| \\
& \leq 2 \mu(A \Delta B) .
\end{aligned}
$$

Thus $\left\{f_{n}\right\}$ is uniformly continuous with respect to $\mu$, and the conclusion of the proposition follows from Proposition 1 .

3.2. Strong Necessity of Zero Entropy. Consider for the moment the special case in which $\mathcal{X}=[0,1)$ is the unit interval equipped with its Borel subsets $\mathcal{B}$, and $\mu$ is Lebesgue measure. As above, let $\Phi$ be the set of invertible measure preserving transformations of $([0,1), \mathcal{B}, \mu)$ endowed with the weak topology. Let $E_{1}, E_{2}, \ldots$ be a countable sequence of 
measurable sets generating $\mathcal{B}$. Define the distance between transformations $\phi, \psi \in \Phi$ by

$$
d(\phi, \psi)=\sum_{i=1}^{\infty} \frac{1}{2^{i}}\left[\mu\left(\phi E_{i} \triangle \psi E_{i}\right)+\mu\left(\phi^{-1} E_{i} \triangle \psi^{-1} E_{i}\right)\right] .
$$

It follows from standard results [9] that $(\Phi, d)$ is a complete metric space, and that the topology generated by $d(.,$.$) coincides with the weak topology on \Phi$.

Proposition 3. Let $T$ be an invertible ergodic measure preserving transformation of the space $([0,1), \mathcal{B}, \mu)$ and let $\mathcal{C} \subseteq \mathcal{B}$ be a family of Borel sets. If $H(\mathcal{C})>0$, then there exists $\delta>0$ such that

$$
G_{N}(T, \delta)=\left\{\phi \in \Phi: \sup _{n \geq N} \sup _{C \in \mathcal{C}} \int\left|\frac{1}{n} \sum_{i=0}^{n-1} I_{C}\left(T_{\phi}(x)\right)-\mu(C)\right| d \mu(x)>\delta\right\} .
$$

is dense in $\Phi$ for every $N \geq 1$.

Proof: By Lemma 3, there exists $\delta_{0}>0$ such that, for every finite measurable partition $\Pi$ of $[0,1)$ there is some set $C \in \mathcal{C}$ such that $H(C \mid \pi)>\delta_{0}$. It then follows from Lemma 1 that for every finite measurable partition $\Pi$

$$
\mu\left(\cup\left\{A \in \Pi: \delta_{1} \mu(A) \leq \mu(A \cap C) \leq\left(1-\delta_{1}\right) \mu(A)\right\}\right)>\delta_{1}
$$

for some constant $\delta_{1}>0$ depending only on $\delta_{0}$. Define $\delta=\delta_{1}^{3} / 64$. Fix $N \geq 1$ and let $\epsilon>0$ and $\phi \in \Phi$ be given. We will construct invertible measure preserving transformations $S, \psi$ and $\tau$ such that the following three conditions hold

$$
S_{\psi}=T_{\phi \tau^{-1} \psi}, \quad d\left(\phi, \phi \tau^{-1} \psi\right)<\epsilon, \quad \text { and } \sup _{n \geq N} \sup _{C \in \mathcal{C}} \int\left|\frac{1}{2 n} \sum_{i=0}^{2 n-1} I_{C}\left(S_{\psi}^{i} x\right)-\mu(C)\right| d \mu>\delta .
$$

To this end, choose integers $n \geq N$ and $m \geq 1$ such that

$$
\frac{16}{n}<\epsilon \text { and } \quad \sum_{i=m}^{\infty} \frac{1}{2^{i}}=\frac{1}{2^{m-1}}<\frac{\epsilon}{2} .
$$

Let $D_{0}, D_{1}, \ldots, D_{2 n-1} \subseteq[0,1)$ be a Rohklin tower of height $2 n$ for $T_{\phi}$ : in other words, the sets $D_{i}$ are disjoint and satisfy the relations

$$
D_{i}=T_{\phi}^{i} D_{0} \quad 0 \leq i \leq 2 n-1 \text { and } \mu\left(\bigcup_{i=0}^{2 n-1} T_{\phi}^{i} D_{0}\right)>1-\frac{1}{2 n} .
$$

Let $E_{1}, E_{2}, \ldots$ be the sequence of generating sets used to define the metric $d(.,$.$) on \Phi$. With $m \geq 1$ as in 5 define finite partitions $\Gamma_{0}=\left[\bigvee_{i=1}^{m} E_{i}\right] \vee\left[\bigvee_{i=1}^{m} \phi^{-1} E_{i}\right]$ and $\Gamma_{1}=\bigvee_{i=0}^{2 n-1} T_{\phi}^{-i} \Gamma_{0}$ of $[0,1)$. Let

$$
\Pi_{0}=\left\{A \cap D_{0}: A \in \Gamma_{1}\right\}=\left\{A_{0}, \ldots, A_{K}\right\}
$$

be the partition of $D_{0}$ induced by $\Gamma_{1}$, and let

$$
\Pi_{1}=\left\{T_{\phi}^{i}\left(A_{k}\right): A_{k} \in \Pi_{0}, 0 \leq i<2 n\right\}
$$


be a partition of the levels of the Rohklin tower obtained from the partition of the base $D_{0}$ using the map $T_{\phi}$. Note that $\tilde{\Pi}_{1}=\Pi_{1} \cup\left\{\left(\cup_{i} D_{i}\right)^{c}\right\}$ is a partition of $[0,1)$. Our choice of $\Gamma_{1}$ ensures that for each $A \in \Pi_{1}$ and each $1 \leq j \leq m$ either $A \subset E_{j}$ or $A \subset E_{j}^{c}$, and either $A \subset \phi^{-1} E_{j}$ or $A \subset \phi^{-1} E_{j}^{c}$. Define sets

$$
F_{j}=\cup\left\{A \in \Pi_{1}: A \subset E_{j}\right\} \text { and } F_{j}^{\prime}=\cup\left\{A \in \Pi_{1}: A \subset \phi^{-1}\left(E_{j}\right)\right\} .
$$

It follows from $(6)$ that $\mu\left(F_{j} \triangle E_{j}\right)<\frac{1}{2 n}$ and $\mu\left(F_{j}^{\prime} \triangle \phi^{-1} E_{j}\right)<\frac{1}{2 n}$ for each $1 \leq j \leq m$.

Choose $C \in \mathcal{C}$ such that $H\left(C \mid \tilde{\Pi}_{1}\right)>\delta$. Let $\beta_{0}=0$ and define $\beta_{k}=\sum_{l=0}^{k-1} \mu\left(A_{l}\right)$ for $1 \leq k \leq K$. Note that for each $0 \leq k \leq K$ the set $A_{k}$ is isomorphic to the interval $\left[\beta_{k}, \beta_{k+1}\right)$. For each $k$ define an invertible measure preserving map $\psi_{k}: A_{k} \rightarrow\left[\beta_{k}, \beta_{k+1}\right)$ such that

$$
\psi_{k}\left(C \cap A_{k}\right)=\left[\beta_{k}, \beta_{k}+\mu\left(C \cap A_{k}\right)\right)
$$

Let $\alpha_{0}=0$ and define $\alpha_{i}=\sum_{l=0}^{i-1} \mu\left(D_{i}\right)=i \mu\left(D_{0}\right)$ for $1 \leq i \leq 2 n-1$. Define $\psi: D_{0} \rightarrow\left[0, \alpha_{1}\right)$ such that $\psi(x)=\psi_{k}(x)$ for $x \in A_{k}$. Extend $\psi$ to an invertible measure preserving map on $[0,1)$ as follows:

$$
\begin{aligned}
\psi: D_{i} & \rightarrow\left[\alpha_{i}, \alpha_{i+1}\right) \\
\psi: T_{\phi}^{i}\left(A_{k}\right) & \left.\rightarrow\left[\alpha_{i}+\beta_{k}, \alpha_{i}+\beta_{k+1}\right)\right) \\
\psi: C \cap T_{\phi}^{i}\left(A_{k}\right) & \rightarrow\left[\alpha_{i}+\beta_{k}, \alpha_{i}+\beta_{k}+\mu\left(C \cap T_{\phi}^{i} A_{k}\right)\right) \\
\psi:\left(\cup_{i} D_{i}\right)^{c} & \rightarrow\left[\alpha_{2 n-1}, 1\right) .
\end{aligned}
$$

Thus $\psi$ maps each set $T_{\phi}^{i}\left(A_{k}\right) \in \Pi_{1}$ to the interval $\left[\alpha_{i}+\beta_{k}, \alpha_{i}+\beta_{k+1}\right)$, and maps the intersection of $C$ and $T_{\phi}^{i}\left(A_{k}\right)$ to the left side of the interval. Define $S:[0,1) \rightarrow[0,1)$ such that

$$
S(x)= \begin{cases}x+\mu\left(D_{0}\right) & \text { if } x \in\left[0, \alpha_{2 n-2}\right) \\ \psi \circ T_{\phi} \circ \psi^{-1}(x) & \text { if } T_{\phi}\left(\psi^{-1} x\right) \in\left(\bigcup_{i=0}^{2 n-1} D_{i}\right)^{c} \\ S^{-2 n+1} \circ \psi \circ T_{\phi}^{2 n} \circ \psi^{-1}(x) & \text { if } T_{\phi}\left(\psi^{-1} x\right) \in D_{0}\end{cases}
$$

One may readily verify that $S$ is invertible and measure preserving. Define $\tau:[0,1) \rightarrow[0,1)$ such that

$$
\tau(x)= \begin{cases}\psi(x) & \text { if } x \in\left(\bigcup_{i=0}^{2 n-2} D_{i}\right)^{c} \\ \left(S^{-i} \circ \psi \circ T_{\phi}^{i}\right)(x) & \text { if } x \in D_{2 n-i-1}, 1 \leq i<2 n\end{cases}
$$

It is easy to show by induction that $\tau$ is well defined on $[0,1)$.

The transformation $\psi$ is defined to map small pieces of the set $C$ to the left side of the subcolumns determined by the $\beta_{k}$ 's. This constructs a new tower, consisting primarily of intervals, such that the transformation $S$ has a poor average on $\psi(C)$, as it maps points up the tower. The map $S$ is defined on the top portion of the tower to be isomorphic to $T_{\phi}$. Thus, the map $S_{\psi}$ has a poor average on $C$, and is isomorphic to $T_{\phi}$. Also, $\psi$ has been defined such that $S_{\psi}$ approximates $T_{\phi}$ in the weak topology. The map $\tau$ is the isomorphism 
used to map $S$ to $T_{\phi}$. In particular, $S_{\psi}=T_{\phi \tau^{-1} \psi}$, and $\phi \tau^{-1} \psi$ is close to $\phi$ in our metric. We give a precise proof of these facts below.

Claim 1: $S_{\tau}=T_{\phi}$.

Proof of Claim 1: We show inductively that $\tau^{-1} \circ S \circ \tau=T_{\phi}$ on $D_{2 n-i-1}$ for $1 \leq i<2 n$. Let $i=1$. Then for $x \in D_{2 n-2}$,

$$
\left(\tau^{-1} \circ S \circ \tau\right)(x)=\left(\tau^{-1} \circ S \circ S^{-1} \circ \tau\right)\left(T_{\phi} x\right)=T_{\phi} x
$$

Suppose now that $\tau^{-1} \circ S \circ \tau=T_{\phi}$ for $x \in D_{2 n-i-1}$, and consider $x \in D_{2 n-i-2}$. The definition of $\tau$ and the inductive hypothesis ensure that

$$
\begin{aligned}
\left(\tau^{-1} \circ S \circ \tau\right)(x) & =\left(\tau^{-1} \circ S \circ S^{-i-1} \circ \tau\right)\left(T_{\phi}^{i+1} x\right) \\
& =\left(\tau^{-1} \circ S^{-i} \circ \tau\right)\left(T_{\phi}^{i+1} x\right) \\
& =\left(T_{\phi}^{-i} \circ T_{\phi}^{i+1}\right)(x) \\
& =T_{\phi} x .
\end{aligned}
$$

Suppose $x \in\left(\bigcup_{i=0}^{2 n-2} D_{i}\right)^{c}$. Either $T_{\phi}(x) \in\left(\bigcup_{i=0}^{2 n-1} D_{i}\right)^{c}$ or $T_{\phi}(x) \in D_{0}$. If $T_{\phi}(x) \in$ $\left(\bigcup_{i=0}^{2 n-1} D_{i}\right)^{c}$, then

$$
\begin{aligned}
\left(\tau^{-1} \circ S \circ \tau\right)(x) & =\left(\tau^{-1} \circ S\right)(\psi x) \\
& =\tau^{-1} \circ \psi \circ T_{\phi} \circ \psi^{-1}(\psi x) \\
& =\tau^{-1}\left(\psi\left(T_{\phi} x\right)\right)=T_{\phi} x .
\end{aligned}
$$

If $T_{\phi}(x) \in D_{0}$, then

$$
\begin{aligned}
\left(\tau^{-1} \circ S \circ \tau\right)(x) & =\left(\tau^{-1} \circ S\right)(\psi x) \\
& =\tau^{-1} \circ S^{-2 n+1} \circ \psi \circ T_{\phi}^{2 n} \circ \psi^{-1}(\psi x) \\
& =\tau^{-1} \circ S^{-2 n+1} \circ \psi \circ T_{\phi}^{2 n}(x) \\
& =\left(T_{\phi}^{-2 n+1} \circ \psi^{-1} \circ S^{2 n-1}\right)\left(S^{-2 n+1} \circ \psi \circ T_{\phi}^{2 n}\right)(x) \\
& =T_{\phi}(x) . \square
\end{aligned}
$$

The transformation isomorphic to $T_{\phi}$ with the required "bad" average is $S_{\psi}$. The previous claim implies $S_{\psi}=\left(T_{\phi}\right)_{\tau^{-1} \psi}=T_{\phi \tau^{-1} \psi}$. Hence, we wish to show that $\phi \circ \tau^{-1} \circ \psi$ is a small perturbation of $\phi$ in our metric space.

Claim 2: $d\left(\phi, \phi \circ \tau^{-1} \circ \psi\right)<\epsilon$. 
Proof of Claim 2: For $x \in \bigcup_{i=0}^{2 n-1} D_{i}$ and $0 \leq k \leq K$ the definitions of $\tau$ and $S$ lead to the following chain of equivalences:

$$
\begin{aligned}
x \in T_{\phi}^{i}\left(A_{k}\right) & \Leftrightarrow \psi(x) \in\left[\alpha_{i}+\beta_{k}, \alpha_{i}+\beta_{k+1}\right) \\
& \Leftrightarrow S^{2 n-i-1}(\psi(x)) \in\left[\alpha_{2 n-1}+\beta_{k}, \alpha_{2 n-1}+\beta_{k+1}\right) \\
& \Leftrightarrow \psi^{-1}\left(S^{2 n-i-1}(\psi(x))\right) \in T_{\phi}^{2 n-1}\left(A_{k}\right) \\
& \Leftrightarrow T_{\phi}^{-2 n+i+1}\left(\psi^{-1}\left(S^{2 n-i-1}(\psi(x))\right)\right) \in T_{\phi}^{i}\left(A_{k}\right)
\end{aligned}
$$

Therefore

$$
\tau^{-1} \circ \psi\left(T_{\phi}^{i} A_{k}\right)=T_{\phi}^{i} A_{k} \text { for } 0 \leq i \leq 2 n-1
$$

This implies that

$$
\psi^{-1} \circ \tau\left(T_{\phi}^{i} A_{k}\right)=T_{\phi}^{i} A_{k} \text { for } 0 \leq i \leq 2 n-1
$$

Let $F_{j}=\cup\left\{A \in \Pi_{1}: A \subset E_{j}\right\}$ be the $\Pi_{1}$-approximation of $E_{j}$ defined above. By the triangle inequality, for $1 \leq j \leq m$,

$$
\begin{aligned}
& \mu\left(\phi\left(E_{j}\right) \triangle \phi \circ \tau^{-1} \circ \psi\left(E_{j}\right)\right) \\
& \quad \leq 2 \mu\left(E_{j} \triangle F_{j}\right)+\mu\left(F_{j} \triangle \tau^{-1} \circ \psi\left(F_{j}\right)\right) .
\end{aligned}
$$

It was shown above that $\mu\left(E_{j} \triangle F_{j}\right)<(2 n)^{-1}$. Moreover, (8) implies that

$$
\mu\left(F_{j} \triangle \tau^{-1} \circ \psi\left(F_{j}\right)\right)=0
$$

and hence $\mu\left(\phi\left(E_{j}\right) \triangle \phi \circ \tau^{-1} \circ \psi\left(E_{j}\right)\right)<4 / n$ for $1 \leq j \leq m$. A similar argument using $F_{j}^{\prime}$ in place of $F_{j}$, and applying $(9)$ shows that $\mu\left(\phi^{-1}\left(E_{j}\right) \triangle \psi^{-1} \circ \tau \circ \phi^{-1}\left(E_{j}\right)\right)<4 / n$ for $1 \leq j \leq m$, and therefore

$$
\sum_{j=1}^{m} 2^{-j}\left[\mu\left(\phi\left(E_{j}\right) \triangle \phi \circ \tau^{-1} \circ \psi\left(E_{j}\right)\right)+\mu\left(\phi^{-1}\left(E_{j}\right) \triangle \psi^{-1} \circ \tau \circ \phi^{-1}\left(E_{j}\right)\right)\right]<\frac{8}{n} .
$$

It follows from the choice of $m$ that $d\left(\phi, \phi \circ \tau^{-1} \circ \psi\right)<\epsilon$, as desired.

Claim 3: $\phi \circ \tau^{-1} \circ \psi \in G_{n}(T, \delta)$. 
Proof of Claim 3: Note that $T_{\phi \tau^{-1} \psi}=S_{\psi}$. Define $\Delta=\cup\left\{A \in \tilde{\Pi}_{1}: \delta_{1} \mu(A) \leq \mu(A \cap C) \leq\right.$ $\left.\left(1-\delta_{1}\right) \mu(A)\right\}$, and let $B=\bigcup_{i=0}^{2 n-1} S_{\psi}^{-i} D_{2 n-1}$. Our choice of $C$ ensures that

$$
\begin{aligned}
\delta_{1}<\int_{[0,1)} I_{\Delta}(x) d \mu & =\int_{B} I_{\Delta}(x) d \mu+\int_{B^{c}} I_{\Delta}(x) d \mu \\
& \leq \sum_{i=0}^{2 n-1} \int_{S_{\psi}^{-i} D_{2 n-1}} I_{\Delta}(x) d \mu+\frac{1}{2 n} \\
& =\int_{D_{0}} \sum_{i=0}^{2 n-1} I_{\Delta}\left(S_{\psi}^{i} x\right) d \mu+\frac{1}{2 n}
\end{aligned}
$$

The inequality above follows from the fact that $\mu\left(B^{c}\right) \leq(2 n)^{-1}$; the final expression follows from a standard change of variables. Define sets

$$
\Delta_{1}=\bigcup_{k=1}^{K} \psi^{-1}\left(\left[\beta_{k}, \beta_{k}+\delta_{1} \mu\left(A_{k}\right)\right)\right) \subset D_{0}
$$

and

$$
\Delta_{2}=\bigcup_{k=0}^{K} \psi^{-1}\left(\left[\beta_{k}+\left(1-\delta_{1}\right) \mu\left(A_{k}\right), \beta_{k+1}\right)\right) \subset D_{0} .
$$

Thus a point $x$ is contained in $\Delta_{1}$ if (and only if) it is contained in a cell $A_{k} \in \Pi_{0}$ and is mapped by $\psi$ to the left $\delta_{1}$ fraction of the interval $\left[\beta_{k}, \beta_{k+1}\right)$ associated with $A_{k}$. The set $\Delta_{2}$ has a similar interpretation. Note that $\mu\left(\Delta_{1}\right)=\mu\left(\Delta_{2}\right)=\delta_{1} \mu\left(D_{0}\right) \leq \delta_{1} / 2 n$.

Recall that $\psi$ maps the intersection of $C$ and the set $T_{\phi}^{i} A_{k}$ into the left part of the interval $\left[\alpha_{i}+\beta_{k}, \alpha_{i}+\beta_{k+1}\right)$. Thus if $S^{i}(\psi(x)) \in \psi(C)$ for some $x \in A_{k} \cap \Delta_{2}$, then $S^{i}(\psi(y)) \in \psi(C)$ for every $y \in A_{k} \cap \Delta_{1}$. It follows that

$$
\int_{A_{k} \cap \Delta_{1}} I_{C}\left(S_{\psi}^{i} x\right) d \mu \geq \int_{A_{k} \cap \Delta_{2}} I_{C}\left(S_{\psi}^{i} x\right) d \mu .
$$

for $1 \leq k \leq K$, and therefore

$$
\int_{\Delta_{1}} I_{C}\left(S_{\psi}^{i} x\right) I_{\Delta^{c}}\left(S_{\psi}^{i} x\right) d \mu \geq \int_{\Delta_{2}} I_{C}\left(S_{\psi}^{i} x\right) I_{\Delta^{c}}\left(S_{\psi}^{i} x\right) d \mu
$$

Note that if $x \in \Delta_{2}$ and $S_{\psi}^{i}(x) \in \Delta$, then $S_{\psi}^{i}(x) \notin C$. Thus

$$
\int_{\Delta_{2}} I_{C}\left(S_{\psi}^{i} x\right) I_{\Delta}\left(S_{\psi}^{i} x\right) d \mu=0
$$

Similarly, if $x \in \Delta_{1}$ and $S_{\psi}^{i}(x) \in \Delta_{1}$, then $S_{\psi}^{i}(x) \in C$, and therefore

$$
\int_{\Delta_{2}} I_{C}\left(S_{\psi}^{i} x\right) I_{\Delta}\left(S_{\psi}^{i} x\right) d \mu=0 .
$$


Together, the last three displays imply that for each $0 \leq i \leq 2 n-1$,

$$
\begin{aligned}
& \int_{\Delta_{1}} I_{C}\left(S_{\psi}^{i} x\right) d \mu-\int_{\Delta_{2}} I_{C}\left(S_{\psi}^{i} x\right) d \mu \\
& \quad \geq \int_{\Delta_{1}} I_{C}\left(S_{\psi}^{i} x\right) I_{\Delta}\left(S_{\psi}^{i} x\right) d \mu-\int_{\Delta_{2}} I_{C}\left(S_{\psi}^{i} x\right) I_{\Delta}\left(S_{\psi}^{i} x\right) d \mu \\
& =\int_{\Delta_{1}} I_{C}\left(S_{\psi}^{i} x\right) I_{\Delta}\left(S_{\psi}^{i} x\right) d \mu \\
& =\int_{\Delta_{1}} I_{\Delta}\left(S_{\psi}^{i} x\right) d \mu
\end{aligned}
$$

Finally, note that if $x \in \Delta_{1} \cap A_{k}$ and $S_{\psi}^{i} x \in \Delta$ then $S_{\psi} y \in \Delta$ for every $y \in A_{k}$. Therefore,

$$
\int_{\Delta_{1}} I_{\Delta}\left(S_{\psi}^{i} x\right) d \mu \geq \frac{\mu\left(\Delta_{1}\right)}{\mu\left(D_{0}\right)} \int_{D_{0}} I_{\Delta}\left(S_{\psi}^{i} x\right) d \mu>\delta_{1}\left(\delta_{1}-\frac{1}{2 n}\right) / 2 n
$$

Averaging over $i=0, \ldots, 2 n-1$, the inequalities (17) and (18) imply that either

$$
\int_{\Delta_{1}}\left|\frac{1}{2 n} \sum_{i=0}^{2 n-1} I_{C}\left(S_{\psi}^{i} x\right)-\mu(C)\right| d \mu>\delta_{1} \frac{\left(\delta_{1}-\frac{1}{2 n}\right)}{4 n}
$$

or

$$
\int_{\Delta_{2}}\left|\frac{1}{2 n} \sum_{i=0}^{2 n-1} I_{C}\left(S_{\psi}^{i} x\right)-\mu(C)\right| d \mu>\delta_{1} \frac{\left(\delta_{1}-\frac{1}{2 n}\right)}{4 n} .
$$

Assume that inequality 19 holds. One may reason from (20) in a similar fashion. By the triangle inequality and an elementary change of variables,

$$
\begin{aligned}
& \int_{\Delta_{1}}\left|\frac{1}{2 n} \sum_{i=0}^{2 n-1} I_{C}\left(S_{\psi}^{i} x\right)-\mu(C)\right| d \mu \\
& \leq \int_{\Delta_{1}}\left|\frac{1}{2 n} \sum_{i=-1}^{2 n-2} I_{C}\left(S_{\psi}^{i} x\right)-\mu(C)\right| d \mu+\frac{1}{2 n} \int_{\Delta_{1}}\left|I_{C}\left(S_{\psi}^{2 n-1} x\right)-I_{C}\left(S_{\psi}^{-1} x\right)\right| d \mu \\
& =\int_{S_{\psi}\left(\Delta_{1}\right)}\left|\frac{1}{2 n} \sum_{i=0}^{2 n-1} I_{C}\left(S_{\psi}^{i} x\right)-\mu(C)\right| d \mu+\frac{1}{2 n} \int_{\Delta_{1}}\left|I_{C}\left(S_{\psi}^{2 n-1} x\right)-I_{C}\left(S_{\psi}^{-1} x\right)\right| d \mu \\
& <\int_{S_{\psi}\left(\Delta_{1}\right)}\left|\frac{1}{2 n} \sum_{i=0}^{2 n-1} I_{C}\left(S_{\psi}^{i} x\right)-\mu(C)\right| d \mu+\frac{\delta_{1}}{2 n^{2}}
\end{aligned}
$$

where in the last step we have used the fact that $\mu\left(\Delta_{1}\right) \leq \delta_{1} \mu\left(D_{0}\right) \leq \delta_{1} / n$. Combining the last display with 190 yields the inequality

$$
\int_{S_{\psi}\left(\Delta_{1}\right)}\left|\frac{1}{2 n} \sum_{i=0}^{2 n-1} I_{C}\left(S_{\psi}^{i} x\right)-\mu(C)\right| d \mu>\frac{\delta_{1}}{4 n}\left(\delta_{1}-\frac{1}{2 n}\right)-\frac{\delta_{1}}{2 n^{2}}>\frac{\delta_{1}}{4 n}\left(\delta_{1}-\frac{3}{n}\right) .
$$


By a similar argument, for $1 \leq i \leq 2 n-1$, we have

$$
\int_{S_{\psi}^{j}\left(\Delta_{1}\right)}\left|\frac{1}{2 n} \sum_{i=0}^{2 n-1} I_{C}\left(S_{\psi}^{i} x\right)-\mu(C)\right| d \mu>\frac{\delta_{1}}{4 n}\left(\delta_{1}-\frac{2 j+1}{n}\right) .
$$

Now choose $r$ such that $\delta_{1} / 8<r / n<\delta_{1} / 4$. Then

$$
\begin{aligned}
& \int_{X}\left|\frac{1}{2 n} \sum_{i=0}^{2 n-1} I_{C}\left(S_{\psi}^{i} x\right)-\mu(C)\right| d \mu \\
& \geq \int_{\bigcup_{j=0}^{r-1} S_{\psi}^{j}\left(\Delta_{1}\right)}\left|\frac{1}{2 n} \sum_{i=0}^{2 n-1} I_{C}\left(S_{\psi}^{i} x\right)-\mu(C)\right| d \mu \\
& =\sum_{j=0}^{r-1} \int_{S_{\psi}^{j}\left(\Delta_{1}\right)}\left|\frac{1}{2 n} \sum_{i=0}^{2 n-1} I_{C}\left(S_{\psi}^{i} x\right)-\mu(C)\right| d \mu \\
& >\sum_{j=0}^{r-1} \frac{\delta_{1}}{4 n}\left(\delta_{1}-\frac{2 j+1}{n}\right) \\
& >r\left(\frac{\delta_{1}^{2}}{8 n}\right)>\frac{\delta_{1}^{3}}{64} . \square
\end{aligned}
$$

It follows that $S_{\psi} \in G_{n}\left(T_{\phi}, \delta\right)$. This establishes Claim 3, and completes the proof of the proposition.

Proposition 4. For each positive integer $N$ and $\delta>0, G_{N}(T, \delta)$ is open in $\Phi$.

Proof: Let $\phi \in G_{N}(T, \delta)$. Then there exists $n \geq N$ and $C \in \mathcal{C}$ such that

$$
\delta^{\prime}:=\left|n^{-1} \sum_{i=0}^{n-1} I_{C}\left(T_{\phi} x\right)-\mu(C)\right|>\delta .
$$

Let $\epsilon>0$ be so small that for each $\psi \in \Phi$ with $d(\psi, \phi)<\epsilon$ one has

$$
\mu(\psi(C) \triangle \phi(C))<\frac{\left(\delta^{\prime}-\delta\right)}{2} \text { and } \mu\left(\psi^{-1} \circ T^{-i} \circ \phi(C) \triangle \phi^{-1} \circ T^{-i} \circ \phi(C)\right)<\frac{\left(\delta^{\prime}-\delta\right)}{2}
$$

for $0 \leq i \leq n-1$. For each such $\psi$ we claim that $\mu\left(T_{\psi}^{-i}(C) \triangle T_{\phi}^{-i}(C)\right)<\delta^{\prime}-\delta$ for $1 \leq i \leq n-1$. To see this, note that by the triangle inequality,

$$
\begin{aligned}
\mu\left(T_{\psi}^{-i}(C) \triangle T_{\phi}^{-i}(C)\right) & =\mu\left(\psi^{-1} \circ T^{-i} \circ \psi(C) \triangle \phi^{-1} \circ T^{-i} \circ \phi(C)\right) \\
& \leq \mu\left(\psi^{-1} \circ T^{-i} \circ \psi(C) \triangle \psi^{-1} \circ T^{-i} \circ \phi(C)\right) \\
& +\mu\left(\psi^{-1} \circ T^{-i} \circ \phi(C) \triangle \phi^{-1} \circ T^{-i} \circ \phi(C)\right) \\
& \leq \mu(\psi(C) \triangle \phi(C))+\frac{\left(\delta^{\prime}-\delta\right)}{2}<\delta^{\prime}-\delta .
\end{aligned}
$$


As a consequence, we have

$$
\begin{aligned}
& \int\left|\frac{1}{n} \sum_{i=0}^{n-1} I_{C}\left(T_{\psi}^{i} x\right)-\mu(C)\right| d \mu \\
& \geq \int\left|\frac{1}{n} \sum_{i=0}^{n-1} I_{C}\left(T_{\phi}^{i} x\right)-\mu(C)\right| d \mu-\int\left|\frac{1}{n} \sum_{i=0}^{n-1} I_{C}\left(T_{\psi}^{i} x\right)-\frac{1}{n} \sum_{i=0}^{n-1} I_{C}\left(T_{\phi}^{i} x\right)\right| d \mu \\
& \geq \delta^{\prime}-\frac{1}{n} \sum_{i=0}^{n-1} \mu\left(T_{\psi}^{-i}(C) \triangle T_{\phi}^{-i}(C)\right) \\
& >\delta^{\prime}-\frac{1}{n} n\left(\delta^{\prime}-\delta\right)=\delta .
\end{aligned}
$$

It follows that $G_{N}(T, \delta)$ contains the ball $\{\psi: d(\phi, \psi)<\epsilon\}$. This completes the proof.

3.3. Proof of Theorem 2, By applying a measure space isomorphism, it is enough to prove the theorem for the case where $\mathcal{X}=[0,1), \mathcal{S}=\mathcal{B}, \mathcal{C} \subseteq \mathcal{B}$, and $\mu$ is Lebesgue measure. By Proposition 3 there exists $\delta>0$, such that $G_{N}(T, \delta)$ is dense for all $N \geq 1$; by Proposition 2 each set $G_{N}(T, \delta)$ is open in $\Phi$. Define $G=\bigcap_{N=1}^{\infty} G_{N}(T, \delta)$. As $(\Phi, d(.,)$.$) is$ complete, it follows from the Baire category theorem that $G$ is dense in $\Phi$. If $\phi \in G$, then the definition of $G_{N}(T, \delta)$ ensures that for each $N \geq 1$, there exists $n \geq N$ and $C_{n} \in \mathcal{C}$ such that

$$
\int\left|\frac{1}{n} \sum_{i=0}^{n-1} I_{C_{n}}\left(T_{\phi}^{i} x\right)-\mu\left(C_{n}\right)\right| d \mu>\delta
$$

It follows that the uniform mean ergodic theorem for $\mathcal{C}$ fails to hold for each transformation $T_{\phi}$ with $\phi \in G$.

3.4. Proof of Theorem 1. Theorem 1 follows immediately from Proposition 2 and Theorem 2 ,

\section{Uniform Strong and Weak Mixing for Zero-Entropy Families}

Proof of Corollary 1: For each $n \geq 1$ define the set function $f_{n}: \mathcal{S}^{2} \rightarrow \mathbb{R}$ by $f_{n}(A, B)=$ $\mu\left(A \cap T^{-n} B\right)-\mu(A) \mu(B)$. Let $A_{1}, A_{2}, B_{1}, B_{2} \in \mathcal{S}$ be measurable sets. Then by standard 
arguments,

$$
\begin{aligned}
f_{n}\left(A_{1}, B_{1}\right)-f_{n}\left(A_{2}, B_{2}\right) \leq & \left|\mu\left(A_{1} \cap T^{-n} B_{1}\right)-\mu\left(A_{2} \cap T^{-n} B_{2}\right)\right| \\
& +\left|\mu\left(A_{1}\right) \mu\left(B_{1}\right)-\mu\left(A_{2}\right) \mu\left(B_{2}\right)\right| \\
\leq & \mu\left(A_{1} \triangle A_{2}\right)+\mu\left(T^{-n} B_{1} \triangle T^{-n} B_{2}\right) \\
& +\left|\mu\left(A_{1}\right) \mu\left(B_{1}\right)-\mu\left(A_{1}\right) \mu\left(B_{2}\right)\right|+\left|\mu\left(A_{1}\right) \mu\left(B_{2}\right)-\mu\left(A_{2}\right) \mu\left(B_{2}\right)\right| \\
\leq & \mu\left(A_{1} \triangle A_{2}\right)+\mu\left(T^{-n}\left(B_{1} \triangle B_{2}\right)\right) \\
& +\mu\left(A_{1}\right) \mu\left(B_{1} \triangle B_{2}\right)+\mu\left(B_{2}\right) \mu\left(A_{1} \triangle A_{2}\right) \\
\leq & 2 \mu\left(A_{1} \triangle A_{2}\right)+2 \mu\left(B_{1} \triangle B_{2}\right) .
\end{aligned}
$$

Thus the sequence $\left\{f_{n}\right\}$ is uniformly continuous. Part (i) of the corollary now follows from Proposition 1 .

Part (ii) of the corollary follows from Theorem 2 and classic results of Blum-Hansen [5]. Let $T$ be any strongly mixing invertible transformation on $(\mathcal{X}, \mathcal{S}, \mu)$. Suppose that $H(\mathcal{C})>0$ and that $\phi \in \Phi$. For $C \in \mathcal{C}$,

$$
\begin{aligned}
& \int\left|\frac{1}{n} \sum_{i=0}^{n-1} I_{C}\left(T_{\phi}^{i} x\right)-\mu(C)\right|^{2} d \mu \\
& =\frac{1}{n^{2}} \sum_{i, j=0}^{n-1} \int\left(I_{C}\left(T_{\phi}^{i} x\right)-\mu(C)\right)\left(I_{C}\left(T_{\phi}^{j} x\right)-\mu(C)\right) d \mu \\
& =\frac{1}{n^{2}} \sum_{i, j=0}^{n-1} \int\left[I_{C}\left(T_{\phi}^{i} x\right) I_{C}\left(T_{\phi}^{j} x\right)-\mu(C)^{2}\right] d \mu \\
& =\frac{1}{n^{2}} \sum_{i, j=0}^{n-1}\left[\mu\left(C \cap T_{\phi}^{j-i} C\right)-\mu(C)^{2}\right] .
\end{aligned}
$$

By Theorem 2 and the Cauchy-Schwartz inequality, there exists a dense $G_{\delta}$ subset $G \subset \Phi$ such that the first integral does not converge to zero uniformly over $C \in \mathcal{C}$, and therefore the final sum does not converge to zero uniformly over $C \in \mathcal{C}$. From this, a routine argument shows that

$$
\limsup _{n \rightarrow \infty} \sup _{C \in \mathcal{C}}\left|\mu\left(C \cap T_{\phi}^{-n} C\right)-\mu(C)^{2}\right|>0
$$

as desired.

4.1. Uniform Weak Mixing. Recall that a measure preserving transformation $T$ on $(\mathcal{X}, \mathcal{S}, \mu)$ is weak mixing (and hence ergodic) if for each pair of measurable sets $A, B \in \mathcal{S}$,

$$
\lim _{n \rightarrow \infty} \frac{1}{n} \sum_{i=0}^{n-1}\left|\mu\left(A \cap T^{-i} B\right)-\mu(A) \mu(B)\right|=0 .
$$


The following corollary characterizes uniform weak mixing in terms of entropy. We omit its proof, which is substantially similar to that of Corollary 1 .

Corollary 2. Let $T$ be a weak mixing transformation of $(\mathcal{X}, \mathcal{S}, \mu)$ and let $\mathcal{C} \subseteq \mathcal{S}$.

(i) If $H(\mathcal{C})=0$, then $T$ is uniformly weak mixing on $\mathcal{C}$, in the sense that

$$
\lim _{n \rightarrow \infty} \sup _{A, B \in \mathcal{C}} \frac{1}{n} \sum_{i=0}^{n-1}\left|\mu\left(A \cap T^{-i} B\right)-\mu(A) \mu(B)\right|=0 .
$$

(ii) Suppose that $T$ is invertible and that $(\mathcal{X}, \mathcal{S}, \mu)$ is Lebesgue. If $H(\mathcal{C})>0$, then there exists a dense $G_{\delta}$ set $G \subset \Phi$ such that for each $\phi \in G$,

$$
\limsup _{n \rightarrow \infty} \sup _{C \in \mathcal{C}} \frac{1}{n} \sum_{i=0}^{n-1}\left|\mu\left(C \cap T_{\phi}^{-i} C\right)-\mu(C)^{2}\right|>0 .
$$

\section{REFERENCES}

[1] Adams, T.M. and Nobel, A.B. (2012) Uniform approximation of Vapnik-Chervonenkis classes. Bernoulli 18:4 1310-1319.

[2] Adams, T.M. and Nobel, A.B. (2010) Uniform convergence of Vapnik-Chervonenkis classes under ergodic sampling. Annals of Probability 38(4)1345-1367.

[3] Adams, T.M. and Nobel, A.B. (2010) The gap dimension and uniform laws of large numbers for ergodic processes. Preprint. arXiv:1007.2964 1

[4] Adams, T.M. and Silva, C.E. (1999) $\mathbb{Z}^{d}$ Staircase actions. Ergodic Theory and Dynamical Systems 19 $837-850$.

[5] Blum, J. R. and Hanson, D. L., On the mean ergodic theorem for subsequences, Bull. Amer. Math. Soc., 66 (1960), 308-311.

[6] Dudley, R.M. (1999) Uniform Central Limit Theorems. Cambridge University Press, Cambridge. MR1720712 (2000k:60050)

[7] Fieldsteel, A. and Friedman, N.A. (1986) Restricted orbit changes of ergodic Zd actions to achieve mixing and completely positive entropy. Erg. Th. E Dyn. Sys 6 505-528.

[8] Halmos, P. (1964) Measure Theory, 9th ed., D. Van Nostrand Company, New York. MR0033869 $(11,504 \mathrm{~d})$

[9] Halmos, P.R. (1956) Lectures on ergodic theory, Vol. 3, Amer Mathematical Society.

[10] Rudolph, D.J. (1978) The second centralizer of a Bernoulli shift is just its powers. Israel Journal of Mathematics 29 (2) 167-178.

[11] Tikhonov, S. V. (2007) A complete metric in the set of mixing transformations. Sb. Math. 198(4):575

[12] van der VaArt, A.W. and Wellner, J.A. (1996) Weak Convergence and Empirical Processes. Springer-Verlag, New York. MR1385671 (97g:60035)

[13] VAn Handel, R.(2013) The universal Glivenko-Cantelli property. Probability Theory and Related Fields 155 911-934.

[14] VAPNiK, V.N. (2000) The nature of statistical learning theory. Second edition. Springer-Verlag, New York. MR1719582 (2001c:68110) 
Terrence Adams is with the Department of Defense, 9161 Sterling Drive, Laurel, MD 20723

Andrew Nobel is with the Department of Statistics and Operations Research, University of North Carolina at Chapel Hill, Chapel Hill, NC 27599-3260

E-mail address: nobel@email.unc.edu 